\title{
Les structures diapiriques du delta du Niger
}

\author{
par JEAN MASCLE*
}

\begin{abstract}
Résumé. - On présente brièvement la structure du delta sous-marin du Niger essentiellement caractérisé par la présence de nombreuses déformations diapiriques. L'origine de ces dernières est également disculée. On compare enfin la région du delta du Niger à celle de la marge Nord du golfe du Mexique où de nombreux diapirs évaporitiques sont bien connus.

A bstract. Marine geophysical profiles off Niger Delta reveal the presence of diapiric slructures beneath the continental margin. The hypothesis of a possible evaporitic layer is presented and discussed; the general structure of the arca is briefly compared to the structure of the northern margin of the gulf of Mexico where well-known evaporitic structures occur along the whole margin.
\end{abstract}

M. J. LABEyRIE : 1) Les diapirs sont-ils loin du talus du socle continental du delta du Niger? 2) N'y aurait-il pas, systématiquement pour tous les bassins à diapirs, un affaissement du socle entre la zone des diapirs et le continent ?

M. J. Mascle : 1) Il n'est pas possible de savoir avec précision où se situe la limite du socle continenlal sous le delta du Niger. I.es diapirs caractérisent l'ensemble de la pente du delta et constituent un escarpement topographique net à la base de celle-ci. Ils sont cependant fort probablement à l'intérieur d'une série sédimentaire reposant sur un socle océanique.

2) Fin ce qui concerne les bassins de l'ouest africain il semble qu'effectivement ils se situent dans les zones qui ont été des grabens de trpe est-africain actuel. I'n affaissement du socle entre continent et zone de diapirs peut se généraliser à la structure des marges continentales. Dans le cas du Delta du Niger il a été proposé une relation direcle entre cette surcharge sédincntaire et un enforcement, de l'ordre de $10 \mathrm{~km}$, de la surface de la lithosphère.

M. Pothft (I.F.P.) : Y a-t-jl eu des analyses de vitesse qui permettraient de trancher entre les deux hypothèses :diapirisme salifère ou dû à des argiles sous compactées?
M. J. Mascle : Le Deltá du Niger et en particulier son plateau continental, a fait lobjet de nombreuses études de la part de compagnies pétrolic̀res. Il existe donc certainement un grand nombre d'analyses de vitesse, peut-ètre mème à l'l.F.P. Je n'ai eu aucune possibilité d'en connaltre les résultats. Quoiqu'il en soit je pense que dans le cas du delta du Niger, comme ailleurs (ex. : le delta du Mississipi) il existe une relation entre diapirisme argileux et diapirisme évaporitique. Le premier est souvent lié au second. Il semble que ce soit les mouvements profonds qui initient la mobilisation de couches sous compactées plus superficielles. Ces dernieres constituent des pscudo diapirs en injectant par exemple les failles de croissance.

* Centre océanologique de Bretagne, B. P. 337, 29273 Brest Cedex. Conlribution no 346 du département scientitique du C.O.B. Exposé oral présenté à la séance du 2 décembre 1974 . 